\title{
Potential Mechanisms Involved in the Anticonvulsant Effect of Walnut Extract on Pentylenetetrazole-Induced Seizure
}

\author{
Majid Asadi-Shekaari ${ }^{a} \quad$ Azam Eslami $^{\mathrm{e}}$ Tajpari Kalantaripour ${ }^{\mathrm{d}}$ \\ Siyavash Joukar ${ }^{\mathrm{b}, \mathrm{c}}$ \\ ${ }^{a}$ Neuroscience Research Center and ${ }^{b}$ Physiology Research Center, Institute of Neuropharmacology, and \\ 'Department of Physiology and Pharmacology, School of Medicine, Kerman University of Medical Sciences, and \\ ${ }^{d}$ Department of Basic Science, Kerman Branch, Islamic Azad University, Kerman, and eDepartment of Biology, \\ Science and Research Branch, Islamic Azad University, Fars, Iran
}

\section{Key Words}

Walnut kernel · Seizure $\cdot$ Rat · Anticonvulsant effect ·

Pentylenetetrazole

\begin{abstract}
Objective: It was the aim of this study to determine the potential effect of walnut kernel extract (WKE) on experimentally induced seizures in rats and to evaluate the role of benzodiazepines and ethosuximide (ESM) within these pathways. Materials and Methods: Male Wistar rats were selected and divided into eight groups. Seizures were evoked by intravenous infusion of pentylenetetrazole (PTZ; $2 \mathrm{mg} / \mathrm{ml}$ / min). In combination with PTZ, animals were treated with vehicle or WKE (100 mg/kg i.p.), with or without cotreatment with either flumazenil (FMZ; $5 \mathrm{mg} / \mathrm{kg}$ i.p.), ESM (150 mg/kg i.p.) or diazepam (DPZ; $0.5 \mathrm{mg} / \mathrm{kg}$ i.p.). Results: WKE administration significantly increased the PTZ dose needed to induce the first myoclonic jerk ( $13.09 \pm 1.29$ vs. $49.71 \pm 12.03$ $\mathrm{mg} / \mathrm{kg} ; \mathrm{p}<0.001)$, decreased the severity of seizure grades and reduced the mortality rate to $0 \%$. FMZ did not significantly reduce the anticonvulsant effect of WKE. The combination of DPZ and WKE showed a synergic anticonvulsant effect, whereas ESM had no significant influence $(p>0.05)$ on the WKE effects. Conclusion: These findings indicated
\end{abstract}

that WKE was effective at reducing seizure severity, at increasing the dose to the first myoclonic jerk and highly efficacious at preventing mortality, because $100 \%$ of animals were protected. It seems that this positive effect could apply through signaling pathways other than benzodiazepinemediated $\gamma$-aminobutyric acid receptors and may at least in part be similar to ESM.

(c) 2014 S. Karger AG, Basel

\section{Introduction}

Epilepsy is one of the most common central nervous system disorders, and uncontrolled seizures increase the comorbidities and the chance of mortality [1]. Antiepileptic drugs only provide symptomatic treatment as they suppress seizures but do not have the ability to cure the disease [2]. There is continual research focusing on new therapeutic approaches to prevent and treat epileptic seizures.

The walnut tree (Juglans regia L.) is cultivated throughout Eastern Asia, Southern Europe, Northern Africa, and the United States of America [3]. The Walnut kernel (WK) accounts for $40-60 \%$ of the nut weight. It has high levels of oil (52-70\%) in which polyunsatu-

\begin{tabular}{ll}
\hline KARGER & $\begin{array}{l}\text { @ 2014 S. Karger AG, Basel } \\
1011-7571 / 14 / 0236-0538 \$ 39.50 / 0 \quad \text { Karger }\end{array}$ \\
E-Mail karger@karger.com & $\begin{array}{l}\text { Thisis an Open Access article licensed under the terms of the } \\
\text { Creative Commons Attribution-NonCommercial 3.0 Un- } \\
\text { ported license (CC BY-NC) (www.karger.com/OA-license), } \\
\text { applicable to the online version of the article only. Distribu- } \\
\text { tion permitted for non-commercial purposes only. }\end{array}$
\end{tabular}

Siyavash Joukar, $\mathrm{PhD}$

Physiology Research Center, Institute of Neuropharmacology and Department of Physiology and Pharmacology, School of Medicine, Kerman University of Medical Sciences PO Box 7616914115, Kerman 761 (Iran)

E-Mail jokar@kmu.ac.ir or sjokar@gmail.com 
rated fatty acids predominate [4]. WKs are enriched with many health-beneficial nutrients, especially $\Omega-3$, $\Omega-6$ and $\Omega-9$ fatty acids that are essential for optimum health [4]. Previously, it was demonstrated that WK pretreatment has anticonvulsant and neuroprotective effects [5]. Other studies have also shown that WK consumption may delay the kindling procedure and attenuates the amygdala-kindled seizures in rats $[6,7]$. Hence, the present study was conducted to evaluate the potential of WK extract (WKE) to suppress pentylenetetrazole (PTZ)-induced seizures in rats, focusing on the $\gamma$-aminobutyric acid (GABA)ergic system as the most important inhibitory pathway in the central nervous system as well as on the ethosuximide (ESM) performance pathway to clarify the probable mechanisms.

\section{Materials and Methods}

Drugs

The walnuts were collected from the Rabor area, Kerman Province, Iran, in September 2010. A voucher specimen was deposited at the herbarium of the Faculty of Pharmacy, Kerman University of Medical Sciences (No. 1401-1). PTZ hydrochloride, diazepam (DPZ), flumazenil (FMZ), ESM and dimethyl sulfoxide (DMSO) were purchased from Sigma Chemical Co. (St. Louis, Mo., USA). PTZ, DPZ and FMZ were dissolved in saline $(0.9 \% \mathrm{NaCl})$ immediately before use.

\section{WK Extract}

A 20-gram sample of Iranian walnuts (J. regia) was homogenized in $50 \mathrm{ml}$ of methanol and centrifuged at $10,000 \mathrm{~g}$ for $5 \mathrm{~min}$. After decanting the liquid, the remaining supernatant was dried under a nitrogen atmosphere to remove methanol, resuspended in $10 \mathrm{ml}$ of $25 \mathrm{mM}$ Tris-HCl, $\mathrm{pH} 7.5$, and filtered through a Millipore $0.22-\mu \mathrm{m}$ syringe-driven filter unit (Millipore, Billerica, Mass., USA). The prepared extract was stored in glass vials at $-20^{\circ} \mathrm{C}$ prior to use.

\section{Animals}

Ninety-six male Wistar rats, 3 months old and weighing 250$300 \mathrm{~g}$, were used in this study. The animals were housed in a temperature-controlled room $\left(20 \pm 2{ }^{\circ} \mathrm{C}\right)$ in groups of 3 per cage and had free access to chow and water. The rats were divided into 8 groups (12 in each) and received drugs as follows: (1) PTZ (control group) $2 \mathrm{mg} / \mathrm{ml}$ intravenous infusion; (2) DMSO (the WKE vehicle, $0.1 \mathrm{ml}$ i.p., $30 \mathrm{~min}$ before PTZ infusion $)+$ saline $(0.1 \mathrm{ml}$ i.p., 10 min before DMSO administration) + PTZ; (3) WKE (100 mg/ kg i.p., 30 min before PTZ infusion) + saline + PTZ; (4) WKE + FMZ (5 mg/kg i.p., 10 min before WKE administration) + PTZ [8]; (5) WKE + ESM (150 mg/kg i.p., 10 min before WKE administration) + PTZ [8]; (6) WKE + DPZ (0.5 mg/kg i.p., 10 min before WKE administration) + PTZ [9]; (7) ESM + PTZ, and (8) DPZ + PTZ.

Animal experiments were performed in accordance with the Ethics Committee Guidelines of Kerman University of Medical Sciences (No. 89/123KA).

Mechanisms of the Anticonvulsant

Effect of Walnut Extract
WKE Dose Selection

A pilot study with 4 different doses of $\operatorname{WKE}(50,100,200$ and $500 \mathrm{mg} / \mathrm{kg}$ ) was done for dose selection. Despite the dose-dependent anticonvulsant effect of WKE, the dose of $100 \mathrm{mg} / \mathrm{kg}$, the minimum dose which showed a significant anticonvulsant effect, was used for the experiment.

\section{Convulsion Test}

To induce convulsion, $30 \mathrm{~min}$ after injection of $100 \mathrm{mg} / \mathrm{kg}$ of WKE and other drugs based on a designed protocol for each group, PTZ was infused using a method described in a previous study [10]. Briefly, the rat was restrained for the insertion of a 22 -gauge angiocath, connected by an appropriate tube to a syringe containing the drug, into the lateral vein of the tail. Then, the rat was released to a plexiglass cage and allowed free movement. The PTZ solution, containing $2 \mathrm{mg} / \mathrm{ml}$ of PTZ, was infused at a constant rate of $1 \mathrm{ml} / \mathrm{min}$ using a syringe pump, and the rat was observed during the infusion period [10]. Convulsion severity was scored as follows: stage 0 , no change in behavior; stage 1, ear and facial twitching; stage 2, isolated myoclonic jerks; stage 3, clonus of the forelimbs, neck and/or head; stage 4, clonus of the forelimbs, neck and/or head with rearing and falling; stage 5, generalized clonic seizures (GCS; without the tonic phase) beginning with running and followed by loss of righting reflex [11]. The onset of the first myoclonic jerk was considered as seizure threshold, and with the onset of generalized tonic-clonic convulsions, the infusion was ended [10]. The durations of the convulsant infusion necessary to observe a seizure threshold and generalized tonic-clonic convulsion were measured. The amount of convulsant agent required for induction of the threshold or clonic convulsions was calculated by the following parameters: concentration of the convulsant in the injected liquid, infusion duration, infusion rate, and animal weight [10]. If animals did not show a seizure and/or clonic convulsion after receiving the $80 \mathrm{mg} /$ $\mathrm{kg}$ of PTZ [9], the PTZ injection was stopped and the threshold and/or clonic dose of PTZ was considered as $100 \mathrm{mg} / \mathrm{kg}$. In addition, animals were excluded from the study if the tail vein angiocath insertion was not successful for intravenous infusion of PTZ, or if leakage of injected fluid occurred under the skin of the animal.

\section{Statistical Analysis}

Threshold values and clonic doses of PTZ are presented as means \pm SEM. Comparisons of threshold and clonic doses of PTZ among different groups were performed using the Kruskal-Wallis test followed by the post hoc Bonferroni test. Severity scores are presented as medians. Differences in the severity of convulsions were determined by the Kruskal-Wallis and the Mann-Whitney test, and in mortality by Fisher's exact test. A p value $<0.05$ was considered statistically significant.

\section{Results}

Threshold doses, i.e. the required values of PTZ for the induction of a first myoclonic jerk, in the different groups are shown in figure 1. DMSO and saline did not have a significant effect on the threshold dose of PTZ ( $p>0.05)$. Pretreatment with WKE significantly increased the threshold dose compared with the PTZ group and the 


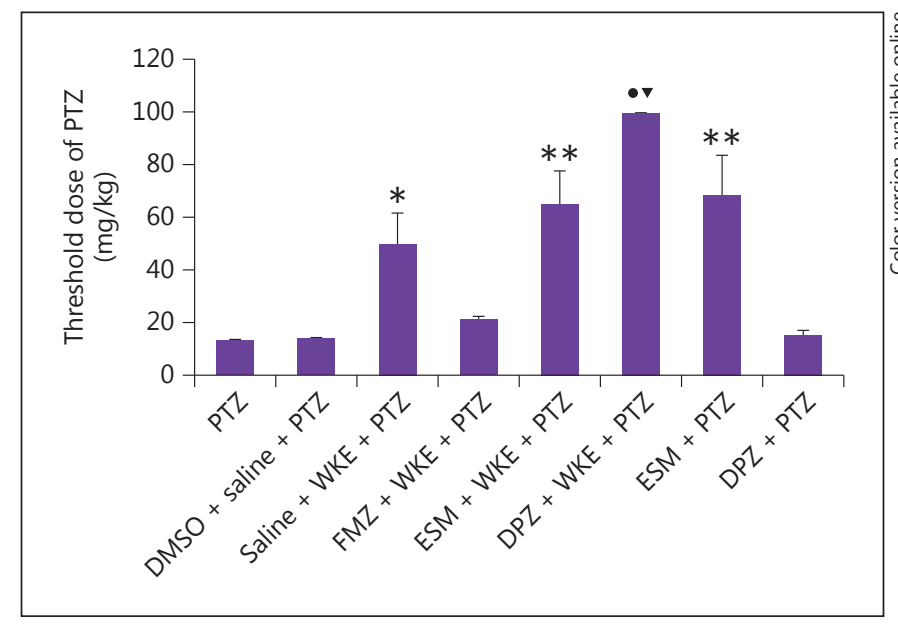

Fig. 1. Required doses of PTZ for the induction of threshold convulsions in the different animal groups. Data are presented as the mean \pm SEM $(n=8-11) .{ }^{*} \mathrm{p}<0.05$ versus the PTZ and DMSO + saline + PTZ groups. ${ }^{* *} \mathrm{p}<0.01$ versus the PTZ, DMSO + saline + $\mathrm{PTZ}$ and DPZ + PTZ groups. ${ }^{\bullet} \mathrm{p}<0.001$ versus the PTZ, DMSO + saline + PTZ, DPZ + PTZ and FMZ + walnut + PTZ groups. ${ }^{\top} \mathrm{p}<$ 0.01 versus the saline + walnut + PTZ group.

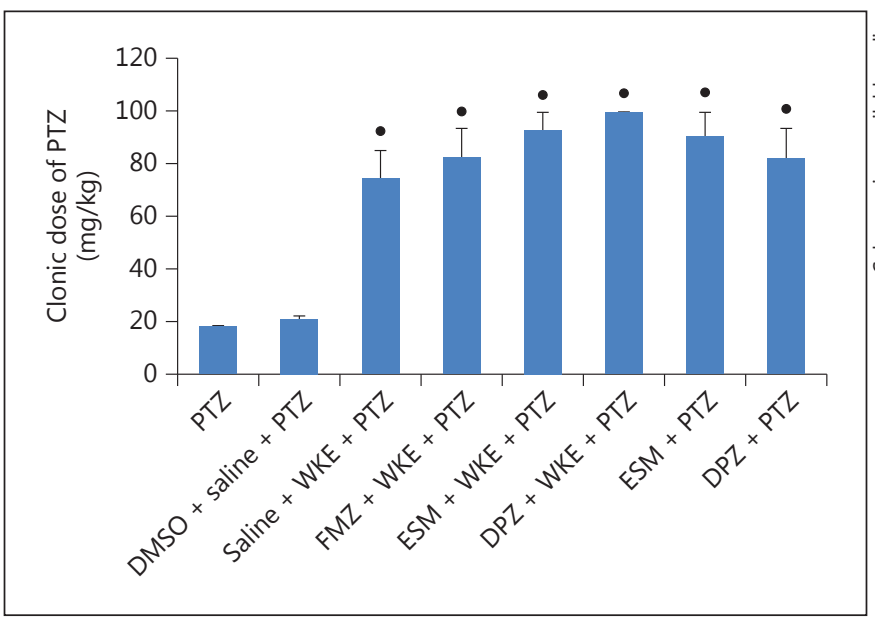

Fig. 2. Required doses of PTZ for the induction of GCS in the different animal groups. Data are presented as the mean \pm SEM $(n=$ $8-11){ }^{\bullet} \mathrm{p}<0.001$ versus the PTZ and DMSO + saline + PTZ groups.

$\mathrm{PTZ}+\mathrm{DMSO}+$ saline group $(\mathrm{p}<0.05)$. Coadministration of WKE with FMZ did not change the threshold dose. However, injection of WKE + DPZ amplified the anticonvulsant effect of WKE and increased the threshold dose ( $\mathrm{p}<0.001$ compared to the PTZ, WKE + PTZ, WKE $+\mathrm{FMZ}+\mathrm{PTZ}$, and PTZ + DZP groups). ESM administration also enhanced the effect of WKE but there was no
Table 1. Convulsion severity and mortality rate in the different animals groups $(n=8-11)$

\begin{tabular}{lcl}
\hline Groups & $\begin{array}{l}\text { Convulsion } \\
\text { severity }\end{array}$ & Mortality \\
\hline PTZ $(\mathrm{n}=9)$ & $5(5-5)$ & $9(100 \%)$ \\
DMSO-saline-PTZ $(\mathrm{n}=9)$ & $5(5-5)$ & $9(100 \%)$ \\
Saline + WKE + PTZ $(\mathrm{n}=11)$ & $4(0-5)^{\mathrm{a}, \mathrm{b}}$ & $0(0 \%)$ \\
FMZ + WKE + PTZ $(\mathrm{n}=8)$ & $3.5(1-5)^{\mathrm{c}, \mathrm{d}}$ & $0(0 \%)$ \\
ESM + WKE + PTZ $(\mathrm{n}=11)$ & $0(0-5)^{\mathrm{e}}$ & $0(0 \%)$ \\
DPZ + WKE + PTZ $(\mathrm{n}=8)$ & $0(0-0)^{\mathrm{f}}$ & $0(0 \%)$ \\
ESM + PTZ $(\mathrm{n}=8)$ & $0(0-5)^{\mathrm{g}}$ & $1(12.5 \%)$ \\
DPZ + PTZ $(\mathrm{n}=8)$ & $4(1-5)^{\mathrm{h}}$ & $2(25 \%)$ \\
\end{tabular}

Seizure severity is expressed as the median, with ranges in parentheses. ${ }^{\mathrm{a}} \mathrm{p}<0.05$ versus the PTZ group. ${ }^{\mathrm{b}} \mathrm{p}<0.05$ versus the $\mathrm{DMSO}+$ saline $+\mathrm{PTZ}$ group. ${ }^{\mathrm{c}} \mathrm{p}<0.05$ versus the $\mathrm{DMSO}+$ saline + PTZ and ESM + walnut + PTZ groups. ${ }^{\mathrm{d}} \mathrm{p}<0.05$ versus the $\mathrm{DMSO}+$ saline $+\mathrm{PTZ}$ group. ${ }^{\mathrm{e}} \mathrm{p}<0.01$ versus the $\mathrm{DMSO}+$ saline + PTZ, FMZ + walnut + PTZ and DPZ + PTZ groups. ${ }^{\mathrm{f}} \mathrm{p}<0.001$ versus the DMSO + saline + PTZ, saline + walnut + PTZ, FMZ + walnut + PTZ and DPZ + PTZ groups. ${ }^{\mathrm{g}} \mathrm{p}<0.01$ versus the DMSO + saline + PTZ, FMZ + walnut + PTZ and DPZ + PTZ groups. ${ }^{\mathrm{h}} \mathrm{p}<$ 0.01 versus the $\mathrm{DMSO}+$ saline $+\mathrm{PTZ}$ and $\mathrm{DPZ}+$ walnut $+\mathrm{PTZ}$ groups.

significant difference between the threshold doses of ESM alone or in combination with WKE (fig. 1).

Administration of WKE significantly increased the PTZ dose for GCS induction ( $<<0.001$ vs. the PTZ group and the PTZ + DMSO + saline group). Moreover, there was no significant difference between the doses of PTZ for GCS in the presence of WKE alone or combined with FMZ or ESM or DZP. In addition, the PTZ dose of GCS in the PTZ + ESM and PTZ + DZP groups in the presence or absence of WKE did not show a significant difference (fig. 2).

The severity of convulsions was significantly reduced by WKE ( $<<0.05$ vs. the PTZ group and the PTZ + DMSO + saline group). This effect of WKE was not affected by FMZ. Furthermore, jerky movements were not seen in the WKE + DZP group at all (table 1).

In this study, PTZ and PTZ + DMSO-induced convulsions were associated with a high mortality rate $(100 \%$ of animals). However, pretreatment with WKE alone or along with FMZ, DPZ and ESM decreased the mortality rate to $0 \%$. In addition, the mortality rate in the ESM + PTZ group and the DPZ + PTZ group was 12.5 and 25\%, respectively. All groups showed significant differences whenever compared with the PTZ and PTZ + DMSO groups $(\mathrm{p}<0.001$; table 1$)$. 


\section{Discussion}

The results showed that WKE increased the required doses of PTZ for the induction of threshold convulsions and GCS. In addition, WKE significantly reduced the severity of convulsions and completely prevented the deaths caused by seizures. The combined use of WKE and DPZ showed a stronger anticonvulsant effect and increased the dose of PTZ for both threshold convulsions and GCS when compared with WKE or DPZ alone. In addition, the animal group that received a combination of WKE and DPZ did not show jerky movements and all animals survived after seizure induction. Administration of FMZ did not significantly decrease the enhancing effect of WKE on the PTZ threshold dose but did not have a prominent effect on the PTZ GCS dose for seizure induction, seizure severity and mortality rate. On the other hand, similar to DPZ, ESM with or without WKE increased the threshold convulsions and GCS and decreased the convulsion severity and mortality; however, there was no significant difference between the effect of ESM alone or along with walnuts.

The GABAergic system is the most important inhibitory system in the central nervous system, but its function may be disturbed in different conditions [12]; GABA is an inhibitory neurotransmitter that can affect $G_{A B A}$ and $\mathrm{GABA}_{\mathrm{B}}$ receptors [12]. $\mathrm{GABA}_{\mathrm{A}}$ are voltage-gated receptors that act with increasing chloride intracellular influx [13].

PTZ is a noncompetitive antagonist of $\mathrm{GABA}_{\mathrm{A}}$ receptors that acts through the t-butyl-bicyclo-phosphorothionate site of the receptor and decreases its activity [14]. Another possibility of PTZ action is to change the potassium and calcium channel conductance [15]. DPZ, as a benzodiazepine receptor agonist [16], can increase the conduction of chloride ion through $\mathrm{GABA}_{\mathrm{A}}$ receptors and induce the anticonvulsant effect [17]. ESM can decrease the conduction of calcium ion from T-type calcium channels and thereby show its anticonvulsant effect [18]. According to the low impact of FMZ as a benzodiazepine receptor antagonist [16] on WKE effects and the synergistic effect of DPZ and walnuts in this study, it is possible that the major anticonvulsant effects of WKE applies through receptors other than the benzodiazepine pathway. On the other hand, the pattern of interaction between the effects of ESM and WKE on the control of seizures raises the possibility that at least part of the anticonvulsant mechanisms of these agents may be similar.

Other pathways may contribute to the anticonvulsant effects of WKE. There is an increasing number of differ-

Mechanisms of the Anticonvulsant

Effect of Walnut Extract ent studies regarding the role of nitric oxide (NO) in the pathophysiology of disorders such as stroke, trauma and seizure disorders $[19,20]$ that could help to explain the anticonvulsant effect of WKE. In epilepsy, NO is also considered an essential pathogenic factor and may have a function in the mechanisms underlying seizure induction and progression [21]. Consistent with this, impressive (five-fold) elevations in NO production were found for the duration of the seizures induced by PTZ. The levels of secondary products resulting from lipid peroxidation have also been shown to be significantly increased in the cerebral cortex of rats with PTZ-induced seizures [22]. A recent study confirmed that walnut extracts diminished the production of NO, tumor necrosis factor- $\alpha$ as well as the expression of inducible NO synthase in BV-2 microglial cells activated by lipopolysaccharide [23]. In another study, primary fatty acids were found to be able to suppress NO production in macrophages [24]. In addition, a recent study has revealed that fatty acids decrease NO production in macrophages stimulated by lipopolysaccharide through iNOS protein expression [25]. WKE and its component ellagic acid have also been shown to possess anti-inflammatory activity in human aorta endothelial cells and osteoblastic activity in KS483 cell lines [26]. In addition, it is reported that excessive production of free radicals has been implicated in the pathogenesis of some neurological disorders, including epilepsy, and it has been suggested that antioxidants as adjuncts to antiepileptic drugs may be helpful for better seizure control [27]. Walnuts contain the highest total level of antioxidants, including both free antioxidants and antioxidants bound to fiber [28]. Therefore, part of the anticonvulsant effect of WKE observed in the present study may mediate through mechanisms that modulate NO production, impede the proinflammatory process and also inhibit the redox imbalance.

\section{Conclusion}

Our findings show the anticonvulsant effect of WKE and suggest that this effect could be exerted through routes other than the involvement of the benzodiazepine action pathway. Activation of the ESM function pathway, reduction in brain NO production, activation of anti-inflammatory mechanisms and reinforcement of the antioxidant system are possible paths of action for WKE in the control of seizures. However, further studies are needed to elucidate the exact mechanisms in which WKE attenuates the PTZ-induced seizures. 


\section{Acknowledgments}

We acknowledge the financial support of this study by Kerman University of Medical Sciences and Health Services of Iran. The data presented in this article are from a Master thesis (A. Eslami) written at the Neuroscience Research Center of Kerman University of Medical Sciences.

\section{Disclosure Statement}

The authors declare that they have no conflicts of interests.

\section{References}

1 Banerjee PN, Filippi D, Allen HW: The descriptive epidemiology of epilepsy - a review. Epilepsy Res 2009;85:31-45.

2 Macleod S, Appleton RE: The new antiepileptic drugs. Arch Dis Child Educ Pract Ed 2007; 92:182-188.

3 Labuckas DO, Maestri DM, Perelló M, et al: Phenolics from walnut (Juglans regia L.) kernels: antioxidant activity and interactions with proteins. Food Chem 2008;107:607-612.

4 Martínez ML, Mattea M, Maestri DM: Varietal and crop year effects on lipid composition of walnut (Juglans regia) genotypes. J Am Oil Chem Soc 2006;83:791-796.

5 Asadi-Shekaari M, Kalantaripour TP, Nejad F, et al: The anticonvulsant and neuroprotective effects of walnuts on the neurons of rat brain cortex. Avicenna J Med Biotechnol 2012;4155-4158

6 Harandi S, Abbasnejad M, Mirnajafi-Zadeh J, et al: Effect of walnut (Juglans regia L.) consumption on anticonvulsant low-frequency stimulation in rats. Online J Vet Res 2013;17: 177-187.

7 Harandi S, Abbasnejad M, Mirnajafi-Zadeh J, et al: Inhibitory effects of walnut consumption on amygdala kindling model of epilepsy in male Wistar rats. Online J Vet Res 2013;17 360-369.

8 Ahmadiani A, Mandgary A, Sayyah M: Anticonvulsant effect of flutamide on seizures induced by pentylenetetrazole: involvement of benzodiazepine receptors. Epilepsia 2003;44: 629-635.

-9 Malhotra J, Seth SD, Gupta SK, et al: Adenosinergic mechanisms in anticonvulsant action of diazepam and sodium valproate. Environ Toxicol Pharmacol 1996;1:269-277.

-10 Joukar S, Atapour N, Kalantaripour TP, et al: Differential modulatory actions of $\mathrm{GABA}_{\mathrm{A}}$ agonists on susceptibility to $\mathrm{GABA}_{\mathrm{A}}$ antagonist-induced seizures in morphine dependent rats: possible mechanisms in seizure propensity. Pharmacol Biochem Behav 2011;99: $17-21$.
11 Silveira Duarte F, Marder M, Ademar Hoeller A, et al: Anticonvulsant and anxiolytic-like effects of compounds isolated from Polygala sabulosa (Polygalaceae) in rodents: in vitro and in vivo interactions with benzodiazepine binding sites. Psychopharmacology 2008;197: 351-360.

12 Mody I, Pearce RA: Diversity of inhibitory neurotransmission through $\mathrm{GABA}_{\mathrm{A}}$ receptors. Trends Neurosci 2004;27:569-575.

13 Ben-Ari Y, Gaiarsa JL, Tyzio R, et al: GABA: a pioneer transmitter that excites immature neurons and generates primitive oscillations. Physiol Rev 2007;87:1215-1284.

14 Korpi ER, Gründer G, Lüddens H: Drug interactions at $\mathrm{GABA}_{(\mathrm{A})}$ receptors. Prog Neurobiol 2002;67:113-159.

15 Madeja M, Stocker M, Mushoff V, et al: Potassium currents in epilepsy: effects of the epileptogenic agent pentylenetetrazole on a cloned potassium channel. Brain Res 1994;656:287294.

16 Khan RM, Soliman MR: Effects of benzodiazepine agonist, antagonist and inverse agonist on ethanol-induced changes in beta-endorphin levels in specific rat brain regions. Pharmacology 1993;47:337-343.

17 Macdonald RL, Kelly KM: Antiepileptic drug mechanism of action. Epilepsia 1995;36:2-12.

18 Coulter DA, Hugenard JR, Prince DA: Characterization of ethosuximide reduction of low-threshold calcium current in thalamic neurons. Ann Neurol 1989;25:582-593.

19 Bashkatova V, Vitskova G, Narkevich V, et al: Nitric oxide content measured by ESR-spectroscopy in the rat brain is increased during pentylenetetrazole-induced seizures. J Mol Neurosci 2000;14:183-190.

20 Weisinger H: Arginine metabolism and the synthesis of nitric oxide in the nervous system. Prog Neurobiol 2001;64:365-391.
21 Moazzami K, Emamzadeh-Fard S, Shabani M: Anticonvulsive effect of atorvastatin on pentylenetetrazole-induced seizures in mice: the role of nitric oxide pathway. Fundam Clin Pharmacol 2013;27:387-392.

-22 Bashkatova V, Narkevich V, Vitskova G, et al The influence of anticonvulsant and antioxidant drugs on nitric oxide level and lipid peroxidation in the rat brain during pentylenetetrazole-induced epileptiform model seizures. Prog Neuropsychopharmacol Biol Psychiatry 2003;27:487-492.

23 Willis LM, Bielinski DF, Fisher DR, et al: Walnut extract inhibits LPS-induced activation of BV-2 microglia via internalization of TLR4: possible involvement of phospholipase D2. Inflammation 2010;33:325-333.

24 Ohata T, Fukuda K, Takahashi M, et al: Suppression of nitric oxide production in lipopolysaccharide-stimulated macrophage cells by omega 3 polyunsaturated fatty acids. Jpn J Cancer Res 1997;88:234-237.

25 Aldrige C, Razzak A, Babcock TA, et al: Lipopolysacchride-stimulated RAW 246.7 macrophage inducible nitric oxide synthase and nitric oxide production is decreased by an omega-3 fatty acid emulsion. J Surg Res 2008;149: 296-302.

-26 Papoutsi Z, Kassi E, Chinou I, et al: Walnut extract (Juglans regia L.) and its component ellagic acid exhibit anti-inflammatory activity in human aorta endothelial cells and osteoblastic activity in the cell line KS483. Br J Nutr 2008;99:715-722.

27 Devi PU, Manocha A, Vohora D: Seizures, antiepileptics, antioxidants and oxidative stress: an insight for researchers. Expert Opin Pharmacother 2008;9:3169-3177.

28 Doheny K: Walnuts are top nut for hearthealthy antioxidants. American Chemical Society (Anaheim). WebMD Health News. 2011. http://www.sciencedaily.com/releases/ 2011/03/110327191040.htm' (accessed August $10,2013)$ 